Linguistique, littérature, didactique

\title{
Les problèmes de ponctuation générale soulevés par la poésie contemporaine
}

The general issues of punctuation raised by contemporary poetry

\section{Michel Favriaud}

\section{(2) OpenEdition}

Journals

\section{Édition électronique}

URL : http://journals.openedition.org/pratiques/5101

DOI : 10.4000/pratiques. 5101

ISSN : 2425-2042

\section{Éditeur}

Centre de recherche sur les médiations (CREM)

Référence électronique

Michel Favriaud, «Les problèmes de ponctuation générale soulevés par la poésie contemporaine ", Pratiques [En ligne], 179-180 | 2018, mis en ligne le 31 décembre 2018, consulté le 30 avril 2019. URL http://journals.openedition.org/pratiques/5101; DOI : 10.4000/pratiques.5101

Ce document a été généré automatiquement le 30 avril 2019.

(c) Tous droits réservés 


\title{
Les problèmes de ponctuation générale soulevés par la poésie contemporaine
}

The general issues of punctuation raised by contemporary poetry

\author{
Michel Favriaud
}

1 C'est à partir des années quatre-vingt que la ponctuation est véritablement entrée dans le domaine de la linguistique. Cela ne veut pas dire que des linguistes, comme P. Larousse, H. Sensine ou J. Damourette (sans remonter à ceux du XVIII siècle, de la Renaissance, voire de l'Antiquité), ne s'y soient penchés, mais ils restaient souvent dans la tradition normative des traités, plus énumérative qu'explicative. Ce sont plutôt les écrivains G. Sand en tête - qui, à partir du XIX ${ }^{\mathrm{e}}$ siècle, ont mis au jour les « problèmes » de la norme - en faisant valoir les textes, le style et la vie, inaugurant ainsi ce que F. Rastier (ici même) appelle une « linguistique des œuvres ».

2 Il faudrait étudier de près et raccommoder le va-et-vient fructueux entre linguistique et littérature pour requalifier le champ de la ponctuation et en faire un enjeu majeur de la linguistique de texte (et probablement de la didactique du français - Favriaud, 2014 ; 2016). F. Rastier dans cette livraison le parcourt à grandes enjambées.

3 Venant des courants contemporains de la linguistique elle-même, on peut avancer que la séparation d'une linguistique de l'oral d'une linguistique de l'écrit, peut-être à dépasser aujourd'hui, amena le courant autonomiste, dominant le champ de la ponctuation à partir des années quatre-vingt (Anis, 1983a; Nunberg, 1990) qui coupait la ponctuation de l'oral ; tandis que la théorie orthographique de $\mathrm{N}$. Catach installait à rang égal les signes de ponctuation dans le plurisystème mixte français comme tiroir «idéographique » non coupé de l'oralité (Catach, 1980, 1994 ; Jaffré, 1991), ce qui fit débat entre les deux camps ; que les théories de l'énonciation (É. Benveniste, M. Bakhtine, O. Ducrot, J. Authier-Revuz) déplacèrent le rôle traditionnellement syntaxique dévolu à la ponctuation vers une approche plus discursive, (Védénina, 1980 ; Anis, 1983a ; Dalhet, 2003), ou rythmique (Meschonnic, 1997) ; qu'enfin, la résurgence d'une linguistique diachronique, fondée en 
partie sur la paléographie, permit de mettre au jour le compagnonnage constant entre écriture, textualité et ponctuation depuis plus de cinq mille ans, fût-il encore, avant le traité massorétique du VIII ${ }^{\mathrm{e}}$ siècle, puis la Renaissance, balbutiant, ou intermittent (Tournier, 1980 ; Poccetti, 2011 ; Stutzmann, 2016).

Mais venant de la littérature, une autre révolution se faisait jour en Europe, souvent avec un temps d'avance : ainsi en France par l'importation aux XVIII et $\mathrm{XIX}^{\mathrm{e}}$ siècles de signes comme le tiret à l'occasion de traduction de romans anglais ou allemands (Dessons, 1993), par les audaces typographiques d'un C. Nodier (Christin, 2009) et bien sûr celles plus radicales de $\mathrm{S}$. Mallarmé dans le fameux poème-manifeste Un coup de dés jamais n'abolira le hasard. Le grand mérite de S. Mallarmé utilisant comme métadiscursivement la ponctuation traditionnelle, noire, la mise en page et le blanc, et la typographie différentielle, est d'avoir pré-théorisé la nouvelle syntaxe, le nouveau livre et la nouvelle lecture, qu'il inaugurait par cet acte fondateur. Passant outre l'intuition géniale de N. Catach et R. Laufer (1980), mais laissée sans suite théorique, selon laquelle le blanc, « signe de ponctuation en négatif » était majeur, J. Anis (1983b) et A.-M. Christin (2009) partant de points de vue différents, qui de linguistique autonomiste, qui d'anthropologie et de phénoménologie, vont revisiter S. Mallarmé et $\mathrm{P}$. Reverdy pour montrer l'influence du blanc sur la syntaxe, la sémantique et ce que nous pourrions appeler la scénographie du texte. Ils ouvraient - par la poésie - la voie théorique de notre "ponctuation blanche», et de ce fait la reconstruction du système ponctuationnel en un "plurisystème » (mot jusque-là utilisé par N. Catach pour caractériser l'orthographe), dont les signes ne sont que la partie émergente (Favriaud, $2000 ; 2004 ; 2011 ; 2014 ; 2016$ ). La poésie moderne et contemporaine, dialoguant avec la linguistique des textes et des œuvres, permettrait ainsi, dans le fil de F.de Saussure, É. Benveniste, E. Coseriu, H. Meschonnic, de poser, peut-être à nouveaux frais, non seulement les " problèmes » des signes de ponctuation, des marques de ponctuation comme le blanc et le jeu typographique, et ceux de leurs valeurs, mais aussi ceux, tout aussi capitaux, des unités de discours actualisées par ces diverses marques, car les poèmes, sous leur double régime de vers et de prose, sont à même d'aviver les problèmes de la phrase et de son fonctionnement, du vers comme seconde unité discursive non coïncidente, et donc plus fondamentalement ceux de l'«architecturation » du texte, maintenant prise entre son unité globale, le poème, et ses unités inférieures. Nous passerions ainsi d'une syntaxe phrastique/interphrastique à une syntaxe et à une sémantique plurielles, appelant un nouveau modèle de lecteur, que nous dénommons, par-delà le modèle ternaire de M. Picard (1986), celui du lecteur « créatif » (Favriaud, 2017).

6 Nous essaierons ainsi d'envisager - à l'épreuve de la poésie contemporaine - les nouveaux tiroirs du plurisystème de la ponctuation, blanc, noir et gris, leur rapport avec les unités de discours et la textualité, et quelques fonctionnements simultanés de marques tant au sein de leur unité discursive matricielle que d'autres unités d'accueil, locales ou globale, ce qui pourrait éventuellement interroger et peut-être éclairer un peu autrement la syntaxe et la sémantique plurielles des textes littéraires, et la façon de les lire. 


\section{Pourquoi fallait-il passer du blanc à la ponctuation blanche?}

7 S'il est vrai que le blanc est le « signe » principal, entre les mots, les vers, au retour à la marge par alinéa, avec retrait sortant, rentrant, entre les paragraphes par saut de ligne ou ligne creuse, entre les chapitres par bas de page blanc, par saut de page ou autour du texte-livre, s'il est vrai que le blanc peut aussi s'infiltrer (chez L. Gaspar, A. du Bouchet) à l'intérieur d'un mot, par interlettrage, ou à l'intérieur d'une phrase enjambante entre deux vers ou deux strophes, il mérite d'être étudié en tant que tel, linguistiquement, énonciativement et rythmiquement, au-delà de toute considération décorative ou métaphysique. Ce faisant, nous rejoignons la conception de l'énonciation éditoriale, amorcée par L.G. Védénina (1989), énoncée et promue par E. Souchier (2007) ou M. Arabyan (2016).

D'abord, le blanc est-il un signe? Pas à proprement parler : N. Catach parlant de « signe en négatif ", qui n'a ni graphisme, ni dimension stable, mais dont la substance, contrairement à la définition standard du signe saussurien, fait la valeur. C'est une marque vide, mais prenant forme et taille au contact d'un ou de plusieurs segments (noircis) du texte, et provoquant des fonctionnements et effets de sens tant sur lesdits segments « blanchis » que sur le texte global. Examinons l'avant-dernière page du poèmerécit de M. Loakira (2018).

9 Au-dessous de l'unité globale du poème-livre, nous découvrons toute une hiérarchie d'unités : des unités de chapitres, numérotées, des unités de double page et de page, à blancs contrôlés. Le texte de cette pénultième page est ordinairement blanchi sur quatre faces : les deux marges latérales, le haut de page et le bas de page laissé largement vacant. À l'intérieur de la page ci-après, certains syntagmes sont à leur tour blanchis sur quatre faces, comme le premier vers ou le dernier :

Dégustateur du suave,

l'aigre-doux,

réchauffé,

$\mathrm{du}$

$$
\text { de }
$$

réchauffé,
$\begin{aligned} & \text { l'indigeste } \\ & \text { Tant dispo à m'émouvoir }\end{aligned}$
les rencontres instantanées
$\begin{aligned} & \text { Au seuil du nouvel âge } \\ & \text { à l'épreuve du vertige, } \\ & \text { malaises, }\end{aligned}$
missions



peut-on faire l'hypothèse que plus le segment textuel comporte de faces blanchies, avec un blanc plus épais, plus il devient énonciativement saillant de la continuité linéaire du poème : « dégustateur du suave », « missions » se rapportent tous à la mort évoquée dans les pages précédentes et au mot-titre («Partance »), pour faire sous-titres de page et ainsi entrer potentiellement dans une concaténation alinéaire comme: «Dégustateur du suave, / l'aigre-doux, / missions », qui est bien une autre façon, ambivalente, de qualifier la mort.

11 Le propre de cette syntaxe alinéaire est d'ouvrir le champ des possibles, car les trois segments blanchis sur quatre faces peuvent se relier à d'autres de rang analogue comme «malaise, ", « l'indigeste », « réchauffé » et pourquoi pas «lors» ou encore la suite de monosyllabes. Sachant que l'ordre de concaténation de tous ces mots n'est guère contraint que par, éventuellement, la majuscule (ponctuation grise) de "Dégustateur ", qui semble désigner ce mot comme tête d'énoncé, et par la ponctuation noire, ici avec virgules, sans point, qui semble indiquer un suspens. Toutefois les virgules, hors de leur phrase d'accueil premier, ne semblent peser guère plus que la ponctuation zéro, après « l'indigeste " par exemple. Il appert ainsi que dans cette configuration alinéaire blanchie, la ponctuation noire prend une valeur ancillaire, proche de zéro, dominée qu'elle est par la ponctuation blanche; elle sert, dans la nouvelle structure d'accueil, de balise minimale de séparation. Nous pouvons ainsi esquisser une théorie des intensités ponctuationnelles, avec comme variable non seulement signes et marques, mais unités discursives (Favriaud, 2011).

Revenons aux petits mots qui semblent encercler « l'indigeste » : cette " comète » semble mettre en valeur le syntagme "l'indigeste ", lui-même blanchi sur quatre faces et seule lexie dense de la partie droite du texte. Ici donc ne compteraient pas seulement le nombre de faces blanchies, l'épaisseur du blanc, mais encore le geste («l'indigeste ») poétique graphique et ce contraste lexical entre mot dense et particules et morphèmes lexicaux, qui, dans le cas de "ré" ", deviennent préfixe de lexie, à moins qu'on y retrouve la note musicale de "ré ». Ces morphèmes lexicaux semi-vides font eux aussi une nouvelle concaténation: «de-de-du-lors-des-des-ré », qu'on peut assimiler à un babil enfantin, ou à une destructuration du langage standard, avec ces allitérations en [d], comme dans le mot « dada », souvent attribué au Maroc méridionnal à la nourrice noire.

13 Nous avons peut-être là une base linguistique à une métaphysique du langage, dont se méfiait tant F. de Saussure. Par ailleurs, nous avons étudié comment le blanc associé aux points de suspension en début de poème ou de laisse (strophe) d'A. du Bouchet, pouvait convoquer l'amont du langage, la préparation de la parole, qui pourraient avoir un rapport avec l'endophasie, c'est-à-dire une génétique du langage, telle que la poésie peut la figurer (Favriaud, 2014) et qui intéresse de plus en plus, semble-t-il, les sciences du langage.

$\mathrm{Au}$ loin la parole - les lèvres, qu'elle timbre, l'imaginent : ouverte comme, à vélo quand une pente est dévalée, le froid - soudain le froid qui se traverse -ravivera, en passant, quelque chose de la crudité de l'herbe sciée. (Du Bouchet 1979, n.p.)

La ponctuation blanche, avec ses marques de différents empans, a ainsi actualisé le poème-page et à l'intérieur de celui-ci des « unités fluctuantes » (ibid.) gouvernées par des structurations alinéaires (que nous pourrions appeler syntaxes extraphrastiques), et même deux langages mis en regard: l'un alinéaire, lexical, ordonné sur le mode de la 
parataxe, l'autre alinéaire non-lexical, purement morphématique, et d'une certaine manière péri-sémantique.

\section{au centre, comme ponctuation blanche à rang égal, obligeant la ponctuation noire à se décentrer et redéfinir à son tour. Mais avant d'en entamer la démonstration, place à la ponctuation grise, à bien des égards intermédiaire entre les deux, par ses marques, son fonctionnement et sa sémantique. \\ Des signes et marques subsidiaires mis au rang de ponctuation grise} résiduelles en les intégrant dans un ensemble ponctuationnel élargi :

From the point of view of function, punctuation must be considered together with a variety of other graphical features of the text, including font-and-face alternations, capitalization, indentation and spacing, all of which can be used to the same sorts of purposes. From here on, I will talk about all these graphical devices as instances of text-category indicators of written language ${ }^{1}$.

Nous franchirons un pas supplémentaire en leur attribuant un tiroir autonome de "ponctuation grise", dont le critère premier sera évidemment celui des marques distinctes.

Il ne s'agit pas non plus, à proprement parler, de signes, autonomes, mais plutôt d'une écriture modifiée, par usage des ressources de police, de taille, de couleur, rejoignant ce qu'on appellerait en bande dessinée la "graphiation ». Ce sont néanmoins des marques " positives». La majuscule devrait entrer dans cette catégorie; au début d'un texte ou 
d'un chapitre, au Moyen Âge, elle pouvait se faire lettrine, avec des couleurs et une illustration. Il semblerait logique d'y associer encore le trait de soulignement, traduit au clavier par l'italique, typiquement grise, et probablement l'accolade. Tous mesurent précisément la portée de l'empan, ce que ne faisait pas le blanc. Mais on voit le pont à faire avec les signes doubles d'insertion, guillemets, parenthèses, doubles tirets, qu'on range dans la ponctuation noire - ce qui montre la porosité relative des tiroirs. Le deuxième critère allégué sera le fonctionnement et la valeur.

Des quatre fonctions de base de la ponctuation, segmentation, liaison, saillance, modalisation, on voit que les première et troisième sont les mieux pourvues ici: les marques de ponctuation coupent la ligne discursive et rendent plus visibles les segments grisés. Car ceux-ci fonctionnent sur la ligne, et donc dans le continuum de la phrase et des phrases, et en dehors, actualisant, plus encore que le blanc, de nouvelles concaténations de segments présentant des caractéristiques semblables de police, de taille, de couleur, de soulignement. On pourrait dire que sur la page, voire sur le texte-livre entier, s'établissent ce faisant une ou plusieurs séquences textuelles, partiellement autonomes, de niveau hiérarchique et sémantique potentiellement distinct : on pense aux didascalies de théâtre, souvent en italique. Regardons ce que L. Giraudon (2016, p. 13), qui répond à notre questionnaire en fin de livraison, en fait dans une page de L'Amour est plus froid que le lac:

\author{
la prochaine fois ne vous fatiguez pas \\ envoyez un singe \\ mortifère fragmentation ou vivace variante \\ la Grèce semble s'effondrer \\ les coups de l'Empire perse \\ bien plus que le beurre \\ l'obscurité est grande
}

Les modalités $d u$ dispositif s'apparentent à celles d'un voyage Perte aussi bien que grappes Filles nues en Kodacolor Des antibiotiques et du chocolat On pleure on rit On se souvient puis on oublie Reflet pastel sur l'ensemble des actes Prenez du temps Buvez du schnaps Niquez vos mères Baratez vos cousines et les frères de vos cousines Mangez des moules ou du boudin Les horaires sont restés à la saison d'été

c'est imprimé dessus

\title{
hélas EN AVANT
}

23 Cette troisième page du livre de L.Giraudon, largement blanchie, exclut toute ponctuation noire, mais fait usage de trois marques de ponctuation grise : la majuscule de nom propre et d'entame de phrase dans la «strophe» centrale en italique, l'italique justement et les petites capitales d'imprimerie de l'avant-dernière ligne. On peut faire l'hypothèse discursive que, dans ce cotexte de ponctuation blanche et grise, l'unité phrastique sera peu ou prou remise en cause par d'autres unités émergeantes.

L'expression capitalisée, quoique blanchie sur quatre faces, s'intègre syntaxiquement à la phrase, comme thème appelant prédicat, mais en même temps ressort de celle-ci, faisant une sorte de post-titre, dont la portée s'exerce sur la page, la double page, voire le livre 
entier. L'attribution en est indécise, faute notamment d'aucun autre segment capitalisé dans cette œuvre: s'agit-il d'un slogan ou d'un stéréotype extérieur ici répercutés, ou d'une injonction au lecteur pour qu'il persévère dans la lecture? En tout cas, c'est la mise en scène d'un dialogisme fort, dans la phrase et dans le texte.

L'italique alliée aux majuscules d'entame, elle, délimite une unité discursive de taille supérieure, faite de phrases courtes, souvent nominales, chacune suivie d'un blanc correspondant à plusieurs espaces, l'ensemble s'opposant au texte englobant, autrement blanchi, plus verticalisé, sans majuscule d'entame de phrases. Cette unité grisée rappelle les laisses d'A. du Bouchet, largement blanchies, à l'entour et en dedans. Du point de vue syntaxique et discursif, elle pourrait s'apparenter à ce que nous appelons, à la suite de M. Charolles, une "période interphrastique » (Favriaud, 2010, p. 270-271; 2012, p. 69-70 et suivantes). Pourtant $\mathrm{du}$ point de vue syntaxique et sémantique (cohésion interphrastique et cohérence du tout), la séquence en italique ne semble pas tenir un discours plus cohérent que l'autre, elle semble simplement plus métapoétique ( «les modalités du dispositif »), et grivoise. On pourrait s'aider de la séquence en italique à la page de gauche, en regard; mais la comparaison des deux ne fait que confirmer l'« hétérogénéité montrée », au sens de J. Authier-Revuz.

26 La ponctuation grise serait ainsi utilisée par trois fois comme à l'état pur, sans enjeu sémantique premier: geste de dialogisme externe entre auteur et lecteur, lieu d'une transaction qui suscite toujours une interrogation, métapoétique, métasyntaxique, métadiscursive. La ponctuation grise actualise toujours le fil (ondulé) du discours général, et en même temps le pervertit, l'interroge sur ses sources énonciatives et ses conditions de fabrication et de lecture. En ce sens, elle est bien intermédiaire entre ponctuation blanche et ponctuation noire. Étonnamment, ce geste (méta)poétique, nous l'avons trouvé dans plusieurs textes d'une élève de CE1, utilisant couleurs et tailles de caractère, d'une façon quasi humoristique, et peut-être encore très décorative, mais où poignent les prémisses de la ponctuation et de la littérarité (Favriaud, Dutrait \& Vinsonneau, 2016, p. 166 et sq.). La plupart des maitres aux cycles 2 et 3 de l'école primaire, ne valorisant que la ponctuation noire avec point, point d'interrogation, virgule et guillemets, ne voient pas l'intérêt de cette ponctuation apparemment moins abstraite, plus émotive, alors qu'elle pourrait être un tremplin d'apprentissage et de réflexivité.

Nous voudrions faire un pas supplémentaire vers le monde des enfants, capable lui aussi d'informer et de questionner une théorie linguistique : en posant la question du lien entre ponctuation grise et illustration.

Cette "réiconisation » de l'écriture par la ponctuation grise, nous la retrouvons dans les albums de jeunesse les plus inventifs, comme ceux de B. Poncelet, ici dans Les Cubes (Favriaud). Contentons-nous d'envisager maintenant la double page au lion (Poncelet, 2003, n.p.), qui évoque la maladie d'Alzheimer de la mère de la narratrice : figurent de gauche à droite la tête du lion, yeux bleus, gueule ouverte sur une langue rouge vif, dont la gorge est masquée par un cube en équilibre instable sur lequel est inscrit un énorme « $t$ » noir, en italique grasse ; à droite, le récit littéraire sur fond beige, relatant « la crise de l'ogre ", coupé avant la pénultième ligne par les trois mots : "caillou, beurre, forte ", dans la même police de caractère, grasse, mais de taille majorée, dont l'initiale, le « $c$ » de "caillou », est partiellement masquée; au centre de la double page, le carnet de vie ouvert du personnage-narrateur, donc posé comme antérieur à sa réécriture narrative, avec graphie manuelle et soulignement d'expressions, remplissant quelques vides informationnels dudit récit; enfin dans le dernier tiers droit de la double page, blanche 
sur fond noir, l'inscription de quatre mots en grandes capitales d'imprimerie, séparés par des tirets, le dernier, probablement l'anaphore de "caillou», amputé de ses trois dernières lettres.

La ponctuation grise actualise ainsi par l'écriture alphabétique trois ou quatre séquences distinctes de texte : le récit littéraire, le récit «primitif » de prise de notes manuelle par un personnage, les deux barres de mots formant deux énoncés pathiques bien distincts : « caillou, beurre, forte » plus euphorique, et « caillou-peur-morte-cail » plus dysphorique, enfantin et fragmentaire, peut-être attribuables à la mère folle. Faut-il compter comme une cinquième séquence le cube de la lettre " $t$ », issu peut-être d'une strate enfantine, anthropologique ou métalinguistique, lequel cube entre dans la gueule ouverte du lion, pour une dévoration implicite? Cet icône, associé au cube de la lettre " $t$ », peut apparaitre à son tour comme un simili ponctuant, pathique, voire modal, de lettre, de séquences textuelles, de double page, voire d'album entier. Ce qui nous donne à voir une ontogenèse, anthropologique et poétique, de l'écriture, entre "raison» et «déraison graphique » (Christin, 1995).

On peut ainsi réasserter, grâce à la littérature et la poésie, le rôle polyphonique de la ponctuation grise, sa faculté à mettre en relation des séquences aux instances diverses, prises à la fois dans un ordre linéaire et dans une circulation semi-aléatoire, et avancer maintenant avec un peu plus de fermeté l'hypothèse d'un continuum entre les séquences du texte, la ponctuation (grise) et l'illustration (Favriaud, 2007).

\section{La ponctuation noire et l'intensité potentielle de ses signes}

31 Cette ponctuation noire, trop longtemps assimilée à « la » ponctuation, vue sous l'angle syntaxique, puis syntactico-énonciatif, n'est-elle pas encore présentée aujourd'hui comme un objet (sinon une collection d'objets) abstrait, pure émanation du système alphabétique, remis en cause par A.-M.Christin, et que la notion de «signe" (ponctuationnel) d'une certaine façon, renforce? Le signe linguistique, celui de L. Hjelmslev par exemple, serait sans dimension, taille ni intensité, pure forme sans substance (ou dont la substance serait mise hors champ). Nous voudrions présenter ici, au regard des ponctuations blanche et grise, quelques arguments pour rééquilibrer cette théorie, qui avait l'idéologie de sa langue.

Revenons sur la mignonne "pausette» de J. Damourette (1939), lequel se plaignait, rappelons-le, que la petite "pause» fût marquée inégalement, faute d'un signe spécifique, par la virgule ou rien. On pourrait avancer l'hypothèse contraire que la «montée » irrégulière " au signe » est la trace d'un réglage très fin des intensités, de la ponctuation phonique à signe zéro à la virgule. Notre test portera sur la ponctuation entre sujet nominal et verbe, verbe et complément d'objet direct nominal qui, normalement, serait absente depuis le XVIII ${ }^{e}$ siècle. On aura tous remarqué cependant dans les copies d'étudiants d'aujourd'hui que la virgule du XVIII siècle a fait son grand retour. Le récit-poème en prose, Caméra, d'Édith Azam (2015, p. 125-129), également présente à la fin de la livraison, pourrait nous éclairer :

[1] Plus tard, Oiseau-silex, se cale dans la pierre, dans la paroi rocheuse. Il est, et c'est comme ça, avec sa liberté. Oiseau-silex dans les parois, regarde le lointain, ne tourne pas la tête [...] 
[2] A l'horizon, la muraille se redresse, se hisse sur son socle. Plus glaciale, plus obscène, et les encercle [...]

[3] Caméra, mieux que les autres, mais par défaut, attend imperturbable le signal de rappel. Mieux que les autres: défaut d'espoir. Se concentre uniquement sur les gestes qui lui restent à faire afin de laisser quelques signes pour que l'oubli, au bout du compte, serve un peu : la mémoire [...]

[4] Elle sourit doucement, Caméra, songe que tous ces signes à présent sont sauvés, que Chouette-à-lunes les connaît [...]

[5] Ils savent l'un autant que l'autre que, si légères qu'elles paraissent, les images : déforment la vue. L'un et l'autre ne bougent plus, ils restent dans la même absence. Cela leur prend cent vie d'accepter : le mourir. Caméra hoche la tête, il est l'heure pour elle de se retrouver seule. La mort doit être: une évidence. Oiseau-silex entend, il s'en va. Oiseau-triste. pages P.O.L. semblent contrevenir à la règle de la ponctuation zéro. L'un, en [1] rappelle la pausette de J. Damourette actualisée par une virgule, tandis qu'un cas (en [5]) semble extravagant, puisque le deux-points est probablement le signe de ponctuation noir médian le plus rupteur, et croyons-nous le plus phonique, par accentuation visuophonique. On pourrait se demander si la gamme ne peut être encore augmentée, allant (en [3]) de la virgule au point, et, si l'on peut oser cette expression, un point augmenté par une phrase insérée (" Mieux que les autres : défaut d'espoir »). L'ensemble des phrases (de [3]) forme une période interphrastique, par épexégèse. Deux conclusions provisoires apparaissent ici: dans ce lieu que la norme interdisait de ponctuer, la gamme des ponctuants noirs apparait immense, de la ponctuation zéro au point; d'autre part, la longueur de l'insertion entre sujet et verbe deviendrait à son tour un facteur (ponctuationnel ?) de suspens et d'intensité. Cela nous amène à une hypothèse opposée concernant l'apposition séparant le sujet du verbe, résolue d'une façon simpliste en syntaxe standard par une annulation de la valeur du ponctuant redoublé : une virgule sépare le sujet du verbe, quand deux le raccordent : pour nous, l'apposition opèrerait une double intensification de la césure, d'abord en redoublant le signe, ou en le triplant (en [3]), puis en insérant un segment textuel dont la longueur, variable, est justement un second facteur d'intensité ruptive, et donc de ponctuation.

Nous pourrions alors expliquer l'anomalie de [1] : «Oiseau-silex dans les parois, regarde le lointain » autrement que par un non-respect de la règle d'insertion ponctuationnelle d'un GN de lieu («dans les parois»), avec ponctuants. Visiblement la césure entre «parois » et « regarde » ou plutôt entre le groupe sujet élargi : " Oiseau-silex dans les parois » et le verbe est plus intense que celle entre « Oiseau-silex » et «dans les parois », au point que la première, phoniquement présente, pût être gommée visuellement. Il y a bien ici, de part et d'autre du GN de lieu, une accentuation et une intensité modulée qui suggère que les deux virgules attendues n'auraient pas eu, de toute façon, la même intensité. Muni de ce nouveau passeport on pourrait analyser [4] et faire l'hypothèse que «Caméra ", normalement en apposition à «Elle », comme dislocation de droite, est un sujet potentiel intensifié du verbe suivant, accentué par la virgule intercalée.

Sous cet angle, et contrairement à la doxa, reprise et confortée par exemple par J. Dürrenmatt (2015, p.60), la fin du sujet lexical antéposé au verbe serait toujours ponctuée-accentuée, mais à des degrés différents, du ponctuant phonique (accent de fin de syntagme) à visibilité zéro au ponctuant phonique fort, à visiblité maximale, comme le deux-points, précédé et suivi d'une espace. (Dans la poésie en vers, le contre-rejet du sujet lexical, donc suivi du blanc, serait une autre façon de ponctuer cette frontière). On 
entrerait alors dans une approche guillaumienne de la ponctuation, comme potentielle et actualisable à des degrés divers, au lieu de cette norme intangible, mais de fait toujours contestée, qu'on peut supposer située conventionnellement à un degré moyen de la gradation potentielle. La poésie nous ferait parcourir tous les degrés de l'échelle. En outre, on soumettrait la question de l'apposition entre sujet et verbe à une règle ponctuationnelle et rythmique supérieure, celle de la mise en évidence de la rupture (relative et variable) entre sujet et verbe. Ce qui va, on le pressent, à l'encontre de tous ceux qui considèrent la ponctuation comme un épiphénomène linguistique.

On a vu au passage en [5] qu'un COD et un attribut du sujet, qu'on dit en syntaxe normée insécables du verbe par un ponctuant, l'étaient ici de même par le deux-points. L'insertion, entre verbe et COD ou attribut lexical, de tout autre groupe, pourrait bénéficier de la même analyse, tendant à montrer que l'insertion fait ponctuation, et que la longueur de l'insertion fait intensité (ponctuante). Quand le groupe sujet est long, comprenant des compléments de nom, la virgule ne vient-elle pas séparer, selon une sous-règle générale, groupe-sujet et verbe, contestant déjà la suprématie de la règle linguistique générale, que l'on a vue de toute part attaquée, en poésie particulièrement ? Ne serait-ce pas non plus, sinon une preuve, du moins une présomption que dans la langue écrite, et pas simplement en poésie, l'oral (pas forcément oralisé) n'est pas loin?

En tout cas, si l'on met en regard courriels et travaux des étudiants actuels avec les poèmes contemporains, on voit que les premiers utilisent eux aussi souvent la virgule entre sujet lexical et verbe, ce qui suggèrerait une quasi-certitude et deux hypothèses : 1 ) la poésie nous permet de comprendre des phénomènes que d'autres qualifieraient, sans bienveillance, d'erreurs par ignorance ; 2) la poésie remettrait la question de l'intensité au cœur de la langue-discours, que d'autres approches de langue stricto sensu avaient écartée par principe : en s'appuyant notamment sur la ponctuation étendue ; 3) il y aurait deux sortes de sujet grammatical qu'on confond trop souvent bien que leur emploi diffère : le sujet lexical et le sujet pronominal clitique, réagissant fort différemment aux ponctuants.

\section{Conclusion}

Les poèmes, sans nous donner beaucoup de réponses définitives sur le fonctionnement général de la ponctuation, ni en langue ni dans d'autres discours, permettent toutefois de soulever des "problèmes", au sens d'É. Benveniste (lui-même obnubilé par la «langue poétique » de C. Baudelaire), et peut-être d'envisager l'extension de son champ et de ses fonctions.

39 Le premier effet d'un corpus de poésie en vers, c'est de prendre au sérieux le blanc, sa dimension infiniment variable et la scénographie du texte. Le blanc s'est sémiotisé en ponctuation blanche à partir du moment où la surface d'inscription n'a plus été mesurée à l'aune pratique ou économique, mais à l'aune textuelle, actualisant les unités de discours qu'il faut décrire à tous les niveaux: unités constitutives capables d'insérer, séparer, relier, mettre en relief, modaliser, au sein d'une unité supérieure ayant ellemême sa propre dynamique. Il y a ponctuation blanche, à marque toujours ajustée, parce que le texte ne vit pas seulement de linéarité, que de nouvelles séquences et unités discursives sont mises en circulation au moyen de syntaxes et de sémantiques plurielles, partiellement aléatoires, équilibrées après coup, où le lecteur devient acteur et créateur, comme prédit par S. Mallarmé. 
40 La ponctuation grise, en quittant les bas-fonds de subsidiarité, devient second tiroir de ponctuation à égalité, délimitant précisément, par des moyens graphiques, des segments ou des séquences, à la fois pris dans la ligne comme éléments hétérogènes, et hors de la ligne, raccordant visuellement des éléments spatialement et temporellement dispersés en nouvelles unités plus «fluctuantes » - tour à tour facilitant et inquiétant la lecture : appelant un lecteur agile, rapide ou plus réflexif, questionneur de la polyphonie textuelle. Alors que le blanc, plus abstrait, n'est guère manié par les enfants de bas âge, qui aiment remplir la page, les traits de la ponctuation grise sont expérimentés spontanément par les jeunes scripteurs dès 5-6 ans, entre image et écriture, ce que les maitres souvent négligent et parfois redoutent. Plus encore que la blanche, la grise fait potentiellement rejoindre deux contraires, probablement nécessaires à la littératie: l'émotion et l'imaginaire d'un côté, la réflexivité de type méta de l'autre. En ce sens l'école, à l'instar de la poésie, devrait en faire son jardin de langue.

41 La ponctuation noire apparait alors comme un sous-ensemble de la ponctuation, restreint, quasi-orthographique, valant principalement pour la phrase et ses hypéronymes : période, paragraphe, chapitre, qui ne peuvent plus passer pour le tout de la textualité. Cela ne veut pas dire que la ponctuation noire doive être abstraite de toute émotivité ou intensité : la ponctuation noire modale en ouvrait déjà le chemin. Le point lui-même n'a-t-il pas ses intensités selon le type d'unité qu'il clôt: même si sa taille propre ne varie pas - contrairement à ce que font, et avec quelle jouissance, les élèves de cycle 2 du primaire avant de poser en grosses capitales d'imprimerie le mot FIN, autre ponctuant, celui-ci lexical - l'ampleur du blanc subséquent ne lui offre-t-elle pas sa dimension (Favriaud, 2012, p. 69-72) ? Vice versa, à un lieu donné de la chaine verbale, la ponctuation s'est avérée beaucoup plus variable que ne l'indique la norme, au demeurant tatillonne: y concourent accents phoniques, scalarité des signes retenus en fonction de leur intensité, renforcement des signes par le blanc, jeux d'insertion-suspension lexicale tous éléments de ponctuation élargie, ayant une incidence pragmatique, rythmique, sinon pathique.

42 La ponctuation étendue, que beaucoup de linguistes et de didacticiens encore ignorent (dans une linguistique d'exemples ad hoc, voire même d'extraits de textes - ce qui serait presque impossible dans une « linguistique des œuvres »), apparaitrait ainsi au cœur de la textualité, de la langue et de l'apprentissage comme un actualisateur et un conducteur de premier plan. Elle va jusqu'à reposer les questions de l'iconicité du code écrit, de la variation des normes, des unités discursives, de la syntaxe unique ou multiple, donc de la sémantique plurielle, vue aussi sous l'angle d'une rythmique (ou d'une poétique) de la forme-sens, de la forme-pensée. Les poètes contemporains n'en sont pas souvent les théoriciens, mais les horlogers suisses, comme il sera montré dans l'entretien de six poètes en fin de livraison. Les enfants en difficulté scolaire sont loin d'en ignorer toutes les formes; on pourrait dire qu'ils les expérimentent eux aussi. Certains même y découvrent, soutenus par l'« agir-poétisé » des maitres, qu'apprendre et ponctuer sont l'un et l'autre une question de rythme. 


\section{BIBLIOGRAPHIE}

ANIS, J. (1983a). « Pour une graphématique autonome ». Langue française 59, p. 31-44. En ligne :

https://www.persee.fr/doc/lfr_0023-8368_1983_num_59_1_5164.

ANIS, J. (1983b). «Vilisibilité du texte poétique ». Langue française 59, p. 88-102. En ligne : https://

www.persee.fr/doc/lfr_0023-8368_1983_num_59_1_5167.

ARABYAN, M. (éd.) (2016). «Présentation ». Semen 41, p. 7-25. En ligne : https://

journals.openedition.org/semen/10571.

AZAM, É. (2015). Caméra. Paris : P.O.L.

САTACH, N. (1980). «La ponctuation ». Langue française 45, p. 17-28. En ligne : https://

www.persee.fr/doc/lfr_0023-8368_1980_num_45_1_5260.

CATACH, N. (1994). La Ponctuation. Histoire et système. Paris : Presses universitaires de France.

CHRISTIN, A.-M. (1995). L'image écrite ou la déraison graphique. Paris : Flammarion.

CHRISTIN, A.-M. [2000] (2009). Poétique du blanc. Vide et intervalle dans la civilisation de l'alphabet.

Paris : Vrin.

DALHET, V. (2003). Ponctuation et énonciation. Guyane : Ibis rouge éditions.

DAMOURETTE, J. (1939). Traité moderne de ponctuation. Paris : Larousse.

DESSONS, G. (1993). « Rythme et écriture : le tiret entre ponctuation et typographie ». In : Saint-

Gérand, J.-P. (éd.), Mutations et sclérose. La langue française, 1789-1848. Stuttgart : F. Steiner,

p. 122-134.

DU BOUCHET, A. (1979). L'Incohérence. Paris : Hachette.

DÜRRENMATT, J. (2015). La ponctuation en français. Paris : Orphrys.

FAVRIAUD, M. (2000). La ponctuation. La phrase - dans la poésie contemporaine. Études des œuvres de du Bouchet, Jaccottet, Stéfan. Thèse en littérature française : Université Paris 8.

FAVRIAUD, M. (2004). « Quelques éléments pour une théorie de la ponctuation blanche - à partir de la poésie contemporaine ». L'Information grammaticale 102, p. 18-23. En ligne : https:// www.persee.fr/doc/igram_0222-9838_2004_num_102_1_2559.

FAVRIAUD, M. (2007). « Le continu des ponctuations noire, blanche et des figures colorées - dans la poésie de jeunesse de Siméon-Mellinette ». Champs du Signe (numéro spécial), p. 203-217.

FAVRIAUD, M. (2010). « La phrase déjouée et rejouée dans la poésie contemporaine ». In : Narjoux, C. (éd), La langue littéraire à l'aube du XXI siècle. Dijon : Éditions universitaires de Dijon, p. 269-280.

FAVRIAUD, M. (2011). «Plurisystème ponctuationnel, dimension, intensité des signes et architecturation du texte poétique ». Langue française 172, p. 83-98. En ligne : https:// www.cairn.info/revue-langue-francaise-2011-4-page-83.htm.

FAVRIAUD, M. (2014). Le plurisystème ponctuationnel français à l'épreuve de la poésie contemporaine. Limoges : Lambert-Lucas. 
FAVRIAUD, M. (2016), « Des éléments de ponctuation blanche et de ponctuation grise ». In : Chol, I., Mathios, B. \& Linarès, S. (éds), Livres de poésie. Jeux d'espace. Paris : H. Champion, p. 471-487.

FAVRIAUD, M. (2017). « Ponctuation blanche et grise dans un album de jeunesse de Béatrice Poncelet ». Semen 41, p. 27-50. En ligne : https://journals.openedition.org/semen/10575.

FAVRIAUD, M. \& MIGNON, F. (2012). «La ponctuation dans Juste la fin du monde de Lagarce : une mise en pièce du discours ». In : Richard, É. \& Doquet, C. (dirs), Les représentation de l'oral chez Lagarce. Continuité, discontinuité, reprise. Louvain-la-Neuve :L'Harmattan-Academia, p. 65-86.

FAVRIAUD, M., DUtRAit, C. \& VinSONNEAU, M. (2016) « Co-élaborer la ponctuation au cycle 2 de l'école primaire ». In : Ronveaux, C. \& Léopoldoff, I. (éds), Enseigner les littératures dans le souci de la langue. Berne : P. Lang, p. $\mathrm{xx}-\mathrm{xx}$.

GIRAUDON, L. (2016). L'Amour est plus froid que le lac. Paris : P.O.L.

JAFFRÉ, J.-P. (1991). «La ponctuation du français : études linguistiques contemporaines ». Pratiques 70, p. 61-83. En ligne : https://www.persee.fr/doc/prati_0338-2389_1991_num_70_1_1637.

LAUFER, R. (1980). « Du ponctuel au scriptural (signes d'énoncé et d'énonciation) ». Langue française 45, p. 77-87. En ligne : https://www.persee.fr/doc/lfr_0023-8368_1980_num_45_1_5267. LOAKIRA, M. (2018). Partance. Rabat : Virgule Édition.

MESCHONNIC, H. (1997). « Pour une poétique de la ponctuation ». Le Discours psychanalytique 18, p. 69-72.

NUNBERG, G. (1990). The Linguistics of punctuation. Standford : CSLI.

PICARD, M. (1986). La lecture comme jeu. Essai sur la littérature. Paris : Éd. de Minuit.

POCCETTI, P. (2011). « La réflexion autour de la ponctuation dans l'Antiquité gréco-latine ». Langue française 172, p. 19-35. En ligne : https://www.cairn.info/revue-langue-francaise-2011-4page-19.htm.

PONCELET, B. (2003). Les Cubes. Paris : Éd. Le Seuil.

SOUCHIER, E. (2007). « Formes et pouvoirs de l'énonciation éditoriale ». Communication \& langages 154, p. 23-38. En ligne : https://www.persee.fr/doc/colan_0336-1500_2007_num_154_1_4688.

STUTZMANN, D. (2016). « La ponctuation. Introduction par Dominique Stutzmann ». Les carnetiers de l'IRHT. En ligne : https://irht.hypotheses.org/1916.

TOURNIER, C. (1980). « Histoire des idées sur la ponctuation, des débuts de l'imprimerie à nos jours ». Langue française 45, p. 28-40. En ligne : https://www.persee.fr/doc/

lfr_0023-8368_1980_num_45_1_5261.

VÉDÉNINA, L. G. (1980). « La triple fonction de la ponctuation dans la phrase : syntaxique, communicative et sémantique ». Langue française 45, p. 60-66. En ligne : https://www.persee.fr/ doc/lfr_0023-8368_1980_num_45_1_5265.

VÉDÉNINA, L. G. (1989). Pertinence linguistique de la présentation typographique. Paris : Peeters-Selaf.

\section{NOTES}

1. «Fonctionnellement, la ponctuation peut être considérée de conserve avec beaucoup d'autres marqueurs textuels comme les variations de police et de taille de caractère, l'alinéation et la spatialisation, qui peuvent concourir aux mêmes finalités. Dorénavant, je parlerai de tous ces 
moyens techniques en tant qu'actualisateurs de catégories textuelles dans le champ de l'écrit » [Traduction MF].

\section{RÉSUMÉS}

Cet article vise la double relation entre linguistique et poésie dans le domaine de la ponctuation. Après avoir constaté l'élargissement progressif de la ponctuation au cours des cinq millénaires d'écriture, nous proposons d'examiner les problèmes de linguistique que la poésie contemporaine met sur le devant. Le blanc n'est pas simplement une marque de ponctuation (le signe majeur, selon N. Catach), mais un tiroir distinct du plurisystème ponctuationnel, ayant ses propres unités discursives, syntaxe et sémantique. Il en va de même avec la " ponctuation grise " ou graphique. La ponctuation canonique deviendra alors " ponctuation noire », avec ses signes habituels à valeur tant émotionnelle que métalinguistique. La ponctuation étendue pourrait être analysée comme une scénographie textuelle, éclairant les unités discursives dans leurs mouvements et ajustements pluriels tant de syntaxe que de sémantique. Ainsi la poésie contemporaine nous suggère-t-elle une conception affinée de la ponctuation.

The main target of this article is to show the double relation between linguistics and poetry on the account of punctuation. The historical tendency has been to enlarge punctuation during the five millenaries of writing, which we can continue by examining the problems of linguistics contemporary poetry brings to the fore. Blank is not a simple punctuation grade (the most important, said N. Catach) but a quite distinct drawer of the punctuation plurisystem, with its own discursive units, its syntax and its semantic. The same with grey punctuation (or graphic punctuation). The usual punctuation will be therefore called black punctuation with its usual signs which will be considered as emotional and metalinguistic signs as well. The proposed extended punctuation could be analyzed as a text scenography, able to highlight the different text units, their movements and adjustments combined by a plural syntax at the benefit of a plural semantics. So contemporary poetry suggests a more refined conception of punctuation.

\section{INDEX}

Mots-clés : poésie, plurisystème ponctuationnel, ponctuation blanche, unités discursives, syntaxe plurielle, sémantique plurielle

Keywords : poetry, punctuation plurisystem, white punctuation, discursive units, plural syntax, plural semantics

\section{AUTEUR}

\section{MICHEL FAVRIAUD}

Université Toulouse - Jean Jaurès, LLA-Créatis, EA 4152, F-31000, France 